\title{
Fundamental Characteristics of the Transformation Superplasticity in a Commercially-pure Titanium*
}

\author{
By Norio FURUSHIRO,** Hiroki KURAMOTO,*** \\ Yoshimasa TAKAYAMA*** and Shigenori HORI**
}

\section{Synopsis}

The strain while undergoing allotropic transformation has been considered to be an important component for the transformation superplasticity. The fundamental characteristics of the strain due to a "both ways" passage through the $(\alpha+\beta)$ field under low stress, $\varepsilon_{t r}$ has been investigated for a commercially-pure titanium.

The remarkable strain was found to occur in certain temperature range during heating and also cooling, in which the transformation takes place. The amount of $\varepsilon_{t r}$ was changed by the rate of heating and cooling, $\dot{T}$, showing a maximum at a rate of $0.1 \mathrm{~K} / \mathrm{s}$. The strain generating during heating was always larger than that during cooling in the present study. This has been considered to be attributed to the strain of the transformation itself. The values of $\varepsilon_{t r}$ increased with an increase in the applied stress. It was also shown that the orientation between the stress axis and the rolling direction had an influence on $\varepsilon_{t r}$. This has been explainable qualitatively in terms of both the orientation dependence of $\varepsilon_{t r}$ under stress free condition and also the texture of the specimen.

A possible mechanism for the transformation superplasticity will be discussed on the basis of the stress dependence of $\varepsilon_{t r}$.

Key words: transformation superplasticity; pure titanium; allotropic transformation; temperature cycle.

\section{Introduction}

The strain which generates due to a "both ways" passage through the $(\alpha+\beta)$ field under the application of the low stress, $\varepsilon_{t r}$ is not so large for metallic materials. However, the accumulation of the strain through a number of the temperature cycles, $\sum \varepsilon_{t r}$ results in achievement of an abnormally large strain. This phenomenon is well known as the transformation superplasticity.

Titanium alloys are characterized by their high strength. However, they are regarded, on the other hand, also as difficult materials to work because of the high flow stress and the narrow range of the optimum temperature for the hot working. Therefore, it has been expected that the hot working condition is improved for the titanium alloys. The transformation superplasticity has a probability of the improvement.

While many investigations of the transformation superplasticity have been carried out for iron and its alloys, ${ }^{1-3)}$ a few studies have been made for titanium and its alloys. ${ }^{4-7)}$ In addition, it is not known systematically how $\varepsilon_{t r}$ is influenced by the rate of heat- ing or cooling, the applied stress and also the microstructure under a wide condition of the temperature cycle test.

In this paper, therefore, the relationship between the transformation and the total strain obtained in a temperature cycle, $\varepsilon_{t}$ has been examined first, and then the value of $\varepsilon_{t r}$ is defined and evaluated under various conditions. Finally, the influences of the rate of heating and cooling, the applied stress, and the direction of the stress axis to the rolled direction on the amount of $\varepsilon_{t r}$ have been investigated.

Pure titanium was selected as the specimen of the present study because the result had a very good possibility of providing the fundamentals of the superplasticity for titanium alloys.

\section{Experimental Procedure}

The test mode employed in the present study was compression because it was easy to obtain higher accuracy in measurements of the small displacement and also to quench the specimen quickly after an interruption of the test. The displacement under higher stress than $0.5 \mathrm{MPa}$ was measured in a specially designed compression apparatus (SDCA), while the displacement under lower stress was determined by a thermomechanical analyzer (TMA) of RIGAKU CN8096. Both instruments were used under constant load. The system including a high quality differential transformer was adopted in SDCA, the accuracy of which was within $\pm 2 \mu \mathrm{m}$ of the displacement. The test temperature was regulated by a PID controller in an infrared ray furnace. The main part of SDCA was screened from atmosphere by a transparent quartz tube, which was swept and filled with a purified argon gas. In TMA, a differential dilatometric system in an air was adopted with an alumina bar as the reference specimen. The accuracy of measurement in TMA was $\pm 0.5 \mu \mathrm{m}$.

Two series of the commercially-pure titanium plate, Ti-A and Ti-B, were prepared for specimens. These plates were $6 \mathrm{~mm}$ and about $30 \mathrm{~mm}$ in thickness, respectively. They had been annealed after hot rolling. Chemical compositions of these materials are shown in the Table 1. Cylindrical specimens for compression tests were machined from Ti-A and Ti-B plates

* $\quad$ Based on the paper presented to the 110th ISIJ Meeting, October 1985, S1611, at Niigata University in Niigata. Manuscript received on February 5, 1987; accepted in the final form on May 15, 1987. (C) 1987 ISIJ

** Department of Materials Science and Engineering, Osaka University, Yamadaoka, Suita 565.

*** Formerly Graduate Student of Osaka University. Now at Production Engineering Research Laboratory, Hitachi Ltd., Yoshidamachi, Totsuka-ku, Yokohama 244.

**** Formerly Graduate Student of Osaka University. Now at Department of Mechanical Engineering, Utsunomiya University, Ishiicho, Utsunomiya 321. 
Table 1. Chemical compositions of pure titanium used. (wt. ppm)

\begin{tabular}{cccccc}
\hline & \multicolumn{5}{c}{ Elements } \\
\cline { 2 - 6 } & \multicolumn{1}{c}{$\mathrm{O}$} & $\mathrm{H}$ & $\mathrm{N}$ & $\mathrm{Fe}$ & $\mathrm{Ti}$ \\
\hline Ti-A & 880 & 20.5 & 66 & 580 & bal. \\
Ti-B & 550 & 16 & 32 & 340 & bal. \\
\hline
\end{tabular}

as the stress axis was parallel to the rolling direction, RD. Further specimens were prepared from Ti-B as the stress axis was in the long or the short transverse direction, LT or ST, respectively. All specimens were $5 \mathrm{~mm}$ in diameter, while length was $10 \mathrm{~mm}$ for SDGA and $19 \mathrm{~mm}$ for TMA.

Specimens were heated at rates of 2 and $8.30 \times$ $10^{-2} \mathrm{~K} / \mathrm{s}$ for tests in SDGA and TMA, respectively, followed by holding for $1.2 \mathrm{ks}$ at $1073 \mathrm{~K}$ before all tests of the temperature cycle. Both rates during heating and cooling were set to be equal and constant under each condition. The rate is represented by $\dot{T}$ hereinafter. Values of $\dot{T}$ and the applied stress ranged from 0.01 to $5 \mathrm{~K} / \mathrm{s}$ and from 0 to $1.96 \mathrm{MPa}$, respectively. The range of the temperature cycle was about from 1073 to $1173 \mathrm{~K}$.

\section{Results and Discussion}

1. Relationship between the Strain Occurring during Heating and Cooling and the Phase Transformation

Typical strain-temperature curves obtained are shown in Fig. 1, in which the strain is represented by the nominal value and positive in the compression direction. A remarkable strain is found in a linear part on each curve in Fig. 1, which is seen, for example, from about 1120 to $1150 \mathrm{~K}$ in the curve of $\dot{T}$ of $0.05 \mathrm{~K} / \mathrm{s}$. Optical and electron microstructures in the specimen quenched from several points on the curve of $0.05 \mathrm{~K} / \mathrm{s}$ in Fig. 1 were observed and it was revealed that the steep slope indicated by the arrow mark corresponded to the $\alpha \rightleftarrows \beta$ transformation. ${ }^{8)}$

It is shown in Fig. 1 that the region of the dual phase is about $30 \mathrm{~K}$ wide. The temperature range should depend on the impurity content in the commercially-pure titanium. It is suggested that iron and oxygen have a remarkable effect on the transformation temperature of pure titanium. ${ }^{9} \quad$ Figure 2 shows a phase diagram for $\mathrm{Ti}$-rich region of the $\mathrm{Ti}-$ Fe system, which was reported by Matyka et al. ${ }^{10}$ The $\alpha$ solvus is found to be $1119 \mathrm{~K}$ for the specimen containing iron of $580 \mathrm{ppm}$. On the contrary, oxygen is known as an $\alpha$ stabilizer. Oxygen of 880 ppm is considered to raise the $\alpha$ solvus about $\left.5 \mathrm{~K} .{ }^{9}\right)$ Therefore, the $\alpha$ solvus of the present specimen may be estimated approximately to be $1124 \mathrm{~K}$, while the $\beta$ transus is considered to be about $1160 \mathrm{~K}^{9,10)}$ It is concluded, therefore, that the temperature range of the dual phase region, obtained experimentally, is in good agreement with that estimated on a simple assumption of the effect of iron and oxygen contents on both the $\alpha$ solvus and the $\beta$ transus of the present specimen. These results support strongly an idea that

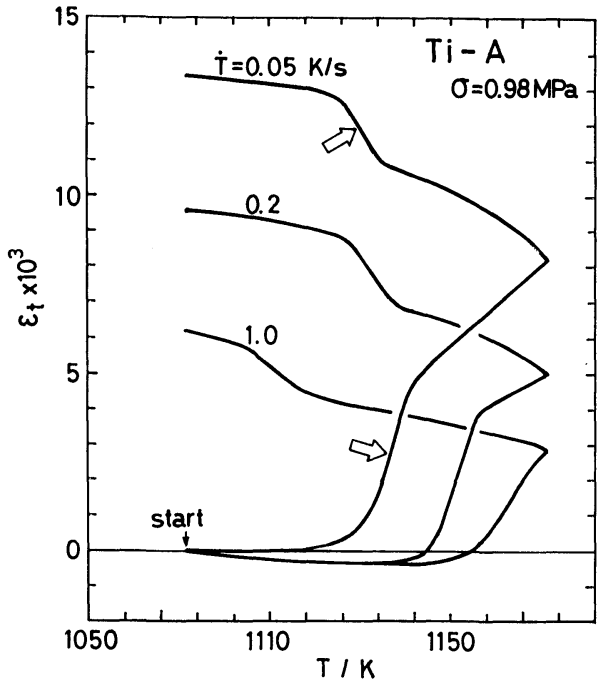

Fig. 1. Typical strain-temperature curves obtained in a temperature cycle under a stress of $0.98 \mathrm{MPa}$ and different rates of heating and cooling for Ti-A.

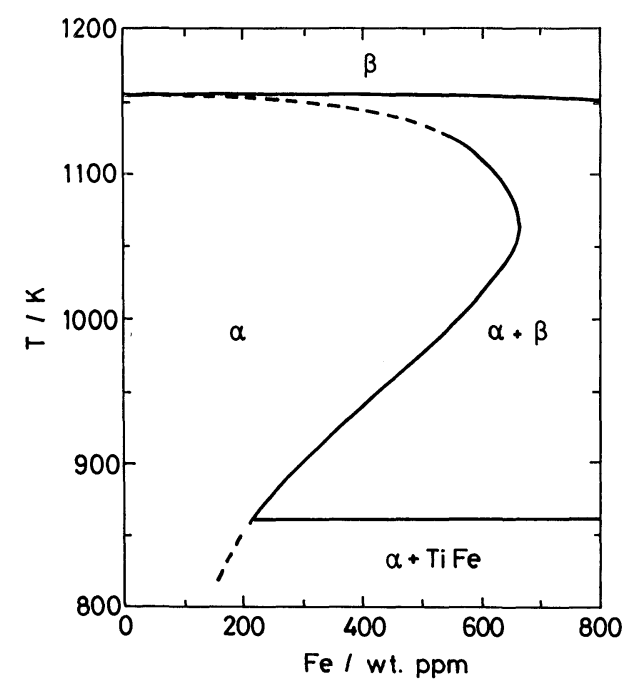

Fig. 2. Titanium rich region of the phase diagram for the $\mathrm{Ti}-\mathrm{Fe}$ system. ${ }^{10}$ )

the commercially-pure titanium should be regarded as a $\mathrm{Ti}-\mathrm{Fe}-\mathrm{O}$ ternary system alloy, especially concerning its phase transformation.

\section{Evaluation of $\varepsilon_{t r}$}

It has been confirmed that the significant strain for the transformation superplasticity appears actually in the steep slope on the strain-temperature curve as shown in Fig. 1. The amount of $\varepsilon_{l r}$ is defined presently as drawn in Fig. 3, where $\varepsilon_{\iota r-l}$ and $\varepsilon_{l r-c}$ correspond to $\varepsilon_{t r}$ during heating and cooling, respectively. The value of $\varepsilon_{t r}$ in the definition is considered to be sum of both strains due to the transformation itself $\varepsilon_{l r(0)}$ and the transformation under the stress $\varepsilon_{l r(\sigma)}$, the latter of which is a characteristic strain for the superplasticity. According to this definition, $\varepsilon_{t r}$ is recognized to be separated from any other strain which involves those due to the thermal expansion and to the creep deformation in a respective single phase region. 


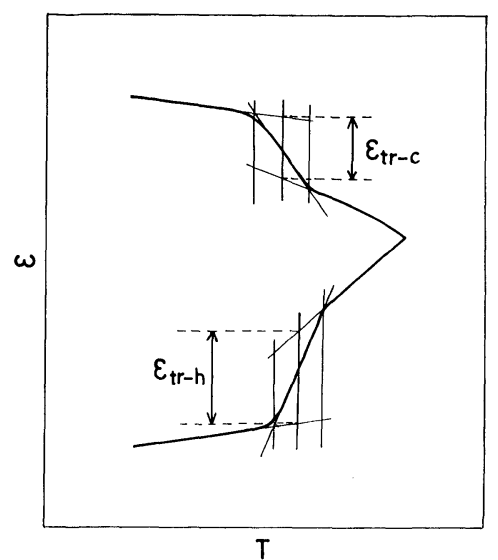

Fig. 3. Definition of $\varepsilon_{t r-h}$ and $\varepsilon_{t r-c}$ on the strain-temperature curve.

\section{Dependence of $\varepsilon_{t r}$ on $\dot{T}$}

In order to examine $\dot{T}$ dependence of $\varepsilon_{t r}$, temperature cycle tests were carried out under conditions of a given stress of $0.98 \mathrm{MPa}$ and of various rates of heating and cooling for one cycle. As seen in Fig. 4, each curve has a maximum at $\dot{T}$ of near $0.1 \mathrm{~K} / \mathrm{s}$. Dependence of $\varepsilon_{t r-c}$ on $\dot{T}$ was similar to that of $\varepsilon_{t r-h}$. The value of $\varepsilon_{t r-c}$ was smaller than the other under all rates examined. The tendency obtained in Fig. 4 may be closely related to periods required to reach the transformation temperature region and also to pass through the region. During the former period, microstructural change such as grain growth may take place under a small $\dot{T}$, while the latter has a reasonably close relation to the amount of $\varepsilon_{t r}$. Therefore, this tendency should be discussed with informations on microstructural and temperature dependence of $\varepsilon_{t r}$. Here, reason for a definite difference between $\varepsilon_{t r-h}$ and $\varepsilon_{t r-c}$ in Fig. 4 is discussed in detail with information on their stress dependence in the next section.

\section{Dependence of $\varepsilon_{t r}$ on the Applied Stress and the Orienta- tion of the Stress Axis to the Rolling Direction of the Specimen}

The relationship between $\varepsilon_{t r}$ and the applied stress for Ti-A under a constant $\dot{T}$ of $0.05 \mathrm{~K} / \mathrm{s}$ is seen in Fig. 5. Values of $\varepsilon_{t r}$ increased with an increase in the stress. The relationship can not be regarded as linear, in contrast to previous results. ${ }^{11-14)}$ Under stress free condition, deviation from the origin to the possitive side (contraction) or the negative side (expansion) takes place in the curves of $\varepsilon_{t r-h}$ or $\varepsilon_{t r-c}$, respectively. These deviations may be attributed to $\varepsilon_{t r(0)}$, as discussed below.

Texture of the rolled or the recrystallized titanium was studied by Keeler and Geisler ${ }^{15}$ ) and Barrett and Massalski. ${ }^{16)}$ For the preferred orientation of the annealed plate after hot rolling, they suggested that [10I0] was oriented in $\pm 14 \sim 20^{\circ}$ from the rolling direction on the rolling plane and also in $\pm 14 \sim 20^{\circ}$ from the transverse direction, and that (0001) pole was oriented in $\pm 25 \sim 40^{\circ}$ from the rolling direction. On the other hand, the Burgers' relation was reported

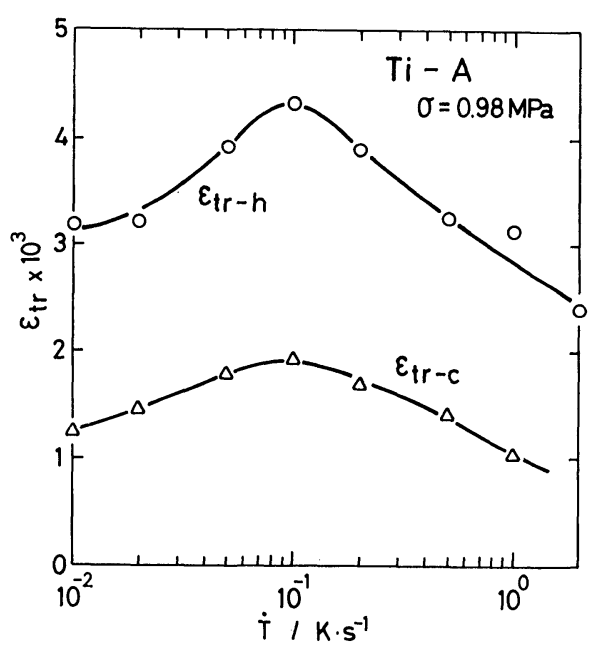

Fig. 4. Influence of $\dot{T}$ on $\varepsilon_{t r-h}$ and $\varepsilon_{t r-e}$ at a stress of 0.98 MPa for Ti-A.

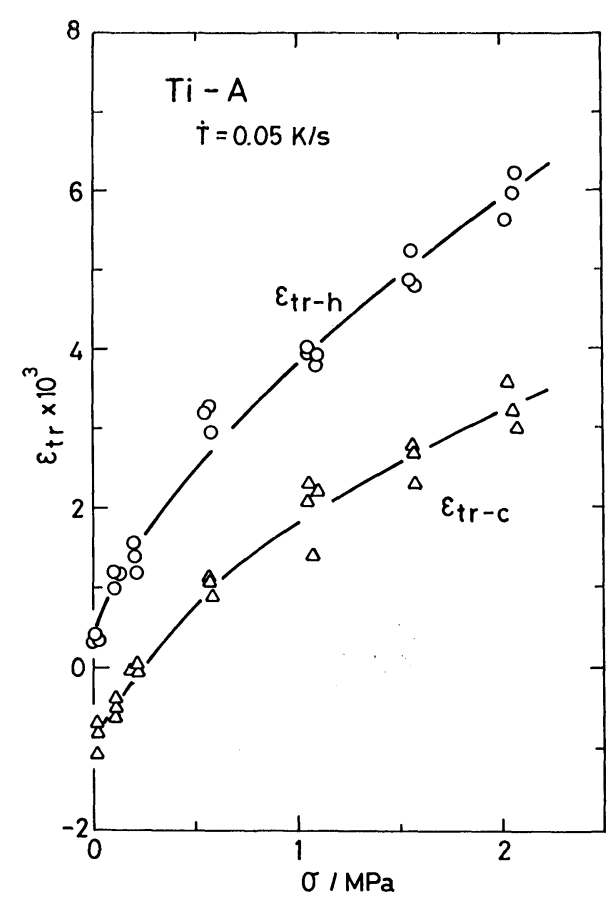

Fig. 5. Influence of the applied stress on $\varepsilon_{t r}$ at a heating and cooling rate of $0.05 \mathrm{~K} / \mathrm{s}$ for Ti-A.

to be held in crystallographic change during the transformation in titanium, which is $\{110\}_{\beta} / /\{0001\}_{\alpha}$ and $\langle 111\rangle_{\beta} / \mid\langle 11 \overline{2} 0\rangle_{\alpha}{ }^{17,18)}$ According to this relation, possible values of $\varepsilon_{t r(0)}$ on simple orientations are estimated as follows, contractions of $0.43 \%$ for $\langle 0001\rangle_{\alpha} \rightarrow\langle 110\rangle_{\beta}$ and $8.8 \%$ for $\langle 1010\rangle_{\alpha} \rightarrow\langle 110\rangle_{\beta}$, and expansion of $11.5 \%$ for $\langle 1120\rangle_{\alpha} \rightarrow\langle 110\rangle_{\beta}$.

Therefore, the presence of both contraction during heating and the expansion during cooling under stress free condition is considered to be explainable qualitatively in terms of both the orientation dependence of $\varepsilon_{t r(0)}$ and the preferred orientation of the specimen.

In order to examine the above concept, influence of the orientation relationship between the stress axis and the rolling direction on $\varepsilon_{t r}$ has been investigated for the thicker plate of Ti-B under the condition of $\dot{T}=0.2 \mathrm{~K} / \mathrm{s}$. Results are shown in Fig. 6, in which 


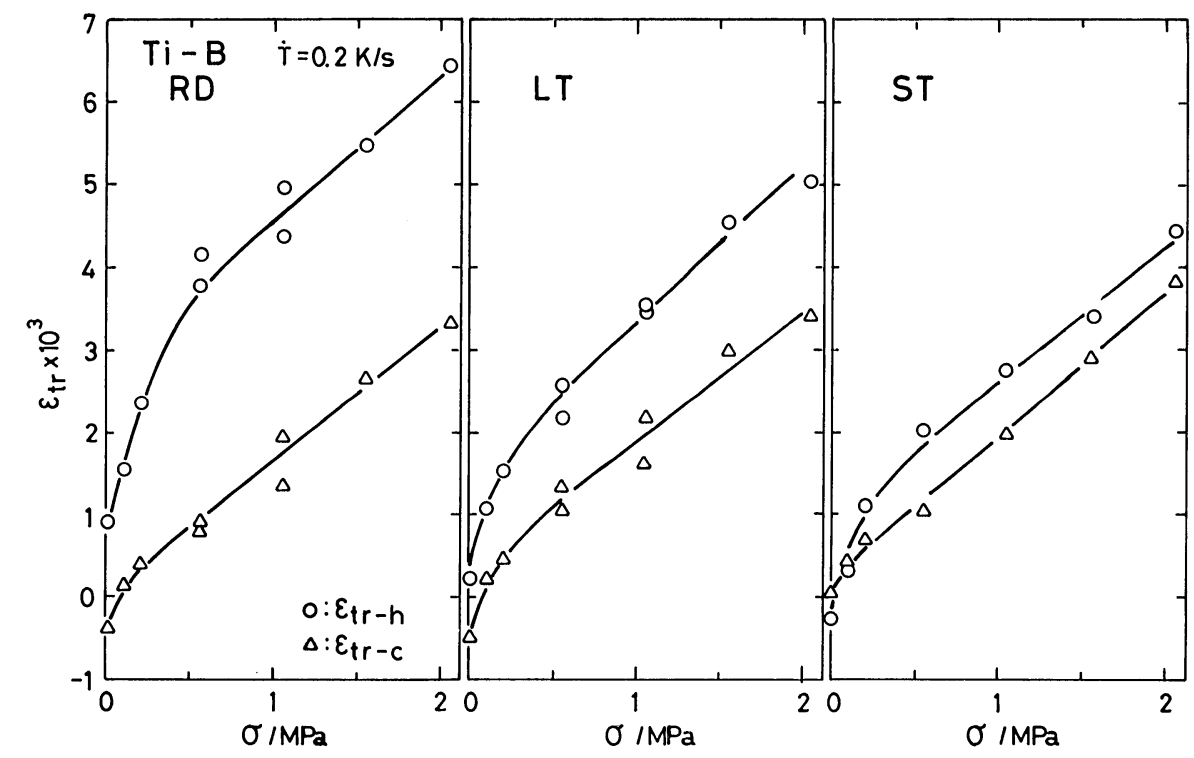

Fig. 6. Influence of the applied stress and its orientation dependence between the stress axis and the rolling direction of the specimen on $\varepsilon_{t r}$ at a heating and cooling rate of $0.2 \mathrm{~K} / \mathrm{s}$ for Ti-B.

the largest $\varepsilon_{t r-h}$ is obtained in RD, while the minimum in ST. On the contrary, it has been found that larger values of $\varepsilon_{t r-c}$ are shown in ST, while the lowest in RD. The difference between them is not so marked, especially in ST, compared with the case of $\varepsilon_{t r-h}$.

A possible reason for these results is considered to be found in the difference of $\varepsilon_{t r(0)}$. During heating of RD and LT, it appears in positive (compression), while in negative (expansion) for ST. By plotting $\varepsilon_{t r(\sigma)}\left(=\varepsilon_{t r}-\varepsilon_{t r(0)}\right)$ against applied stress, Fig. 7 is obtained, showing less difference among RD, LT and ST specimens. It means that the level of $\varepsilon_{t r-h}$ and $\varepsilon_{t r-c}$ is determined mostly by the amount of $\varepsilon_{t r(0)}$. Although the absolute values of $\varepsilon_{t r(0)}$ may be much smaller than the estimated ones mentioned above, the experimental results of orientation dependence of $\varepsilon_{t r(0)}$ are considered to be consistent qualitatively with that estimated. It is still unknown the reason for the quantitative disagreement, but the variety of the microstructure and the different sharpness of the texture may be associated with these discrepancy.

The reason for the stress dependence of $\varepsilon_{t r}$ has been investigated by examination of the stress exponent of the strain rate, $n\left(\dot{\varepsilon}=K^{\prime} \sigma^{n}\right.$, where $\dot{\varepsilon}$ is the strain rate, $\sigma$ the stress and $K^{\prime}$ a constant). The value of $n$ equals to the reciprocal of $m$ which is referred to as a significant parameter for the fine grain superplasticity. ${ }^{19)}$ Presently, the stress can be expressed as follows, $\sigma=$ $K \dot{\varepsilon}_{t r}^{m}$, where $\dot{\varepsilon}_{t r}$ is the strain rate evaluated from the slope on the strain-time curve and $K$ is a constant. Figure 8 shows the relationship between $\dot{\varepsilon}_{t r}$ and stress in double logarithmic plot. The value of $m$ evaluated from the slope was found to be nearly equal to unity. These were consistent with the results reported by Oelschlägel and Weiss ${ }^{20)}$ and Nishihara et al. ${ }^{14)}$ It is very possible that deformation in the transformation superplasticity is controlled by a diffusional process, such as the diffusional creep ${ }^{21-23)}$ and the grain

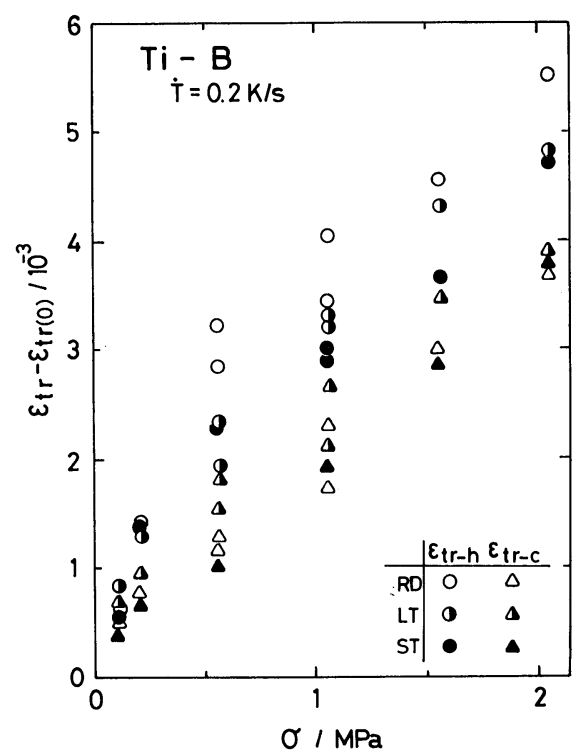

Fig. 7. Relationship between the applied stress and $\varepsilon_{t r(\sigma)}$ $\left(=\varepsilon_{t r}-\varepsilon_{t r(0)}\right)$ for Ti-B.

boundary sliding ${ }^{24,25)}$ at the vicinity of the advancing boundaries during the phase transformation.

\section{Conclusions}

In order to clarify the fundamental characteristics of the transformation superplasticity for a commercially-pure titanium, influences of the heating and cooling rate, the applied stress and the orientation of the stress axis to the rolling direction on the strain while undergoing the transformation for a temperature cycle, $\varepsilon_{t r}$ have been investigated. The results are summarized as follows,

(1) The remarkable strain was found in certain temperature range during heating and also cooling, in which the transformation takes place.

(2) The amount of $\varepsilon_{t r}$ was changed by heating or 


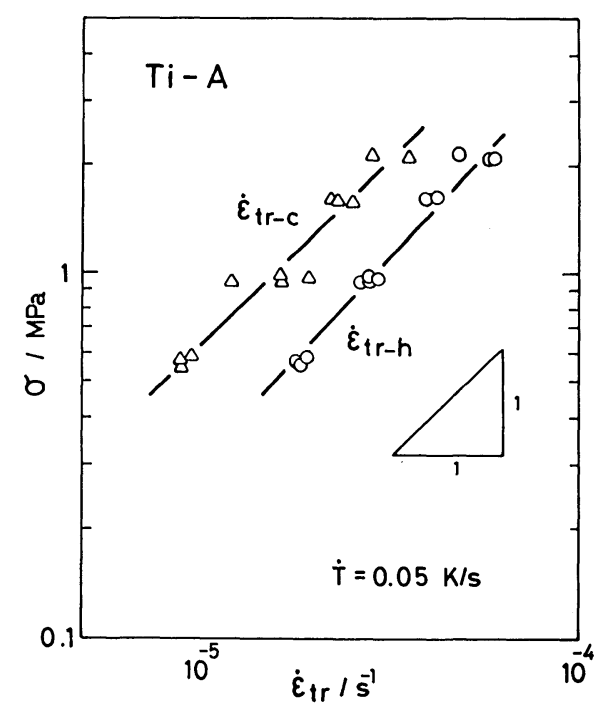

Fig. 8. Relationship between the applied stress and the strain rate during the phase transformation under stresses above $0.5 \mathrm{MPa}$ and a heating and cooling rate of $0.05 \mathrm{~K} / \mathrm{s}$ for $\mathrm{Ti}-\mathrm{A}$.

cooling rate, $\dot{T}$, showing a maximum at a rate of 0.1 $\mathrm{K} / \mathrm{s}$. Values of $\varepsilon_{t r}$ during heating were always larger than those during cooling. This difference has been considered to be attributed to the strain of the transformation itself.

(3) The values of $\varepsilon_{t r}$ during heating and cooling increased with an increase in the applied stress. The orientation between the stress axis and the rolling direction has an influence on $\varepsilon_{t r}$. These are considered to be explainable qualitatively in terms of both the orientation dependence of $\varepsilon_{t r}$ under stress free condition and also the texture of the specimen.

(4) The relationship between the strain rate and the stress while undergoing the allotropic transformation was to be nearly equal to unity. This implies a possibility that the deformation in the transformation superplasticity is controlled by a diffusional process.

\section{Acknowledgements}

The authors are grateful to Drs. Y. Moriguchi and T. Nishimura of Kobe Steel, Ltd. for their efforts in providing the pure titanium. They are also indebted to Mr. Naoto Nishio for his experimental collaboration.

\section{REFERENCES}

1) G. Wasserman: Arch. Eisenhüttenwes., 6 (1933), 347; 10 (1937), 321.

2) N. Iguchi, Y. Oka and Y. Saotome: J. Jpn. Inst. Met., 39 (1975), 457.

3) H. Nozaki, Y. Uesugi, T. Okada and I. Tamura: J. Jpn. Inst. Met., 50 (1986), 56.

4) G. W. Greenwood and R. H. Johnson: Proc. R. Soc. (London), 283A (1965), 403.

5) R. Kot, G. Krause and V. Weiss: Science, Technology and Application of Titanium, Pergamon Press, New York, (1971), 597.

6) T. Nishihara and N. Iguchi: J.Jpn. Inst. Met., 40 (1976), 51.

7) M. Inoue, A. Takahashi, T. Nakasuji, A. Sakamoto and S. Hamai: Tech. Rep. Mitsubishi Heavy Industries Ltd., 14 (1977), 989.

8) Y. Takayama, N. Furushiro and S. Hori: Titanium, Science and Technology, DGM, Oberursel, (1985), 753.

9) M. Hansen and K. Anderko: Constitution of Binary Alloys, McGraw-Hill, New York, (1958).

R. P. Elliott: Constitution of Binary Alloys, 1st Suppl., McGraw-Hill, New York, (1965).

F. A. Shunk: Constitution of Binary Alloys, 2nd Suppl., McGraw-Hill, New York, (1979).

10) J. Matyka, F. Faudot and J. Bigot: Scr. Metall., 13 (1979), 645.

11) M. De Jong and G. W. Rathenau: Acta Metall., 7 (1959), 246.

12) M. De Jong and G. W. Rathenau: Acta Metall., 9 (1961), 714.

13) N. Iguchi, Y. Oka and Y. Saotome: J. Jpn. Inst. Met., 38 (1974), 725

14) T. Nishihara, K. Asami and N. Iguchi: J. Jpn. Inst. Met., 41 (1977), 188.

15) J. H. Keeler and A. H. Geisler: J. Met., 7 (1955), 395; 8 (1956), 80.

16) C. S. Barrett and T. B. Massalski: Structure of Metals, McGraw-Hill, New York, (1966), 576.

17) W. G. Burgers: Physica, 1 (1934), 561.

18) J. B. Newkirk and A. H. Geisler: Acta Metall., 1 (1953), 370.

19) W. A. Backofen, I. R. Turner and D. H. Avery: Trans. Am. Soc. Met., 57 (1964), 980.

20) D. Oelschlägel and D. Weiss: Trans. Am. Soc. Met., 59 (1966), 143.

21) F.R.N. Nabarro: Report of a Conference on Strength of Solids, Phys. Soc., London, (1948), 75.

22) C. Herring: J. Appl. Phys., 21 (1950), 437.

23) R. L. Coble: J. Appl. Phys., 34 (1963), 1679.

24) N. Furushiro and S. Hori: Scr. Metall., 13 (1979), 653.

25) M. F. Ashby, R. Raj and R. G. Gifkins: Scr. Metall., 4 (1970), 737. 